\title{
ON SOLUTIONS OF A NONLINEAR WAVE QUESTION WHEN THE RATIO OF THE PERIOD TO THE LENGTH OF THE INTERVAL IS IRRATIONAL
}

\author{
P. J. MCKENNA
}

\begin{abstract}
The semilinear wave equation $u_{t}-u_{x x}+f(u)=g(t)$ with $u(0, t)=$ $u(\pi, t)=0, u$ is $T$-periodic in $t$, is considered for some situations in which $T$ is not a rational multiple of $\pi$. Various existence results depending on the range of $f^{\prime}$ are given, which contrast sharply with the case where $T$ is a rational multiple of $\pi$.
\end{abstract}

In this paper we consider $T$-periodic solutions of the nonlinear wave equation

$$
\begin{gathered}
u_{t t}-u_{x x}-g(u)=h(x, t), \quad 0<x<\pi, \\
u(0, t)=u(\pi, t)=0, \quad u(x, t)=u(x, t+T),
\end{gathered}
$$

under the assumption that the forcing term $h(x, t)$ satisfies $h(x, t+T)=h(x, t)$. In previous papers $[2,3,6,8]$ this equation has been examined under the assumption that $T$ is a rational multiple of $2 \pi$. The problem of making the transition to $T$ being an irrational multiple of $2 \pi$ has been referred to in [3] as a small divisor problem. The purpose of this paper is to consider some of the phenomena associated with this case. In particular, we demonstrate that the results are totally different from the case where $T=(p / q) \pi$ and $p, q \in Z$.

In $\$ 2$ we restrict ourselves to the case where $T=\pi / \sqrt{2}$. It will be clear from the discussion that these conclusions apply to $T=\pi / \sqrt{n}, n \neq m^{2}$. Here we give a fairly complete description of the phenomena to be expected. In $\$ 3$ we draw some conclusions for a more general class of $\alpha, T=\alpha \pi$. The results proved are of the nonresonance type, which can be found in [2 and 5]. I wish to thank Hugh Montgomery for drawing my attention to references [4 and 7], upon which the results of $\$ 3$ depend. I also wish to thank George Sell for editorial help. The summary of properties of continued fractions was lifted whole from [9].

2. Here we assume that the forcing term $h(x, t)$ satisfies $h(x, t+\pi / \sqrt{2})=h(x, t)$ and we search for weak solutions of the equation

$$
\begin{gathered}
u_{t t}-u_{x x}-g(u)=h(x, t), \quad 0<x<\pi, \\
u(0, t)=u(\pi, t)=0, \quad u(x, t+\pi / \sqrt{2})=u(x, t) .
\end{gathered}
$$

Received by the editors February 4, 1983 and, in revised form, January 16, 1984.

1980 Mathematics Subject Classification. Primary 35J60.

Key words and phrases. Boundary value problems, hyperbolic contractions, compactness. 
We shall consider this as an operator equation on the space $L^{2}(\Omega)$ with $\Omega=\{0, \pi\}$ $\times\{0, \pi / \sqrt{2}\}$. An orthonormal basis for this Hilbert space is

$$
\phi_{j k}^{(1)}=\frac{2 \sqrt{2}}{\pi^{2}} \sin j x \sin \sqrt{2} k t, \quad \phi_{j k}^{(2)}=\frac{2 \sqrt{2}}{\pi^{2}} \sin j x \cos \sqrt{2} k t ;
$$

and if $L u=u_{t t}-u_{x x}$, we have

$$
L \phi_{j k}^{(i)}=\left(j^{2}-2 k^{2}\right) \phi_{j k}^{(i)} .
$$

Thus the spectrum of the operator $L$ with the boundary conditions of (2) is $\sigma(1)=\left\{j^{2}-2 k^{2}, j=1,2, \ldots, k=0,1,2, \ldots\right\}$. We now make a few observations about $\sigma(L)$. Clearly, $0 \notin \sigma(L)$, since $\sqrt{2}$ is irrational, and $\sigma(L)$ is a subset of the integers.

We obtain from [7, \$9.5] that each point of $\sigma(L)$ is an eigenvalue of infinite multiplicity. Indeed, if $k_{1}=M_{1}^{2}-2 N_{1}^{2} \in \sigma(L)$ and $\alpha_{1}+\sqrt{2} \beta_{1}=\left(M_{1}+\sqrt{2} N_{1}\right)^{s}$ for any integer $s$, then $k_{2}=\alpha_{1}^{2}-2 \beta_{1}^{2} \in \sigma(L)$. Thus we have two important differences from the case $T=2 \pi$. The first difference is that $L$ is actually invertible. The second is that there is no decomposition of $L_{2}(\Omega)$ into a part on which $L$ is bounded and a part on which $L^{-1}$ is compact. Thus the method of combining compactness and monotonicity is unlikely to be of much use here.

TheOREM 1. Assume g satisfies:

(i) $|g(x)-g(y)| \leqslant|x-y|, \forall x, y \in R$.

(ii) $|g(x)| \leqslant A|X|+B$ for some constants $A, B, A<1$.

Then equation (2) has a solution for all $h \in L^{2}(\Omega)$.

Proof. Let $B_{R}=\left\{f \in L^{2}(\Omega):\|f\| \leqslant R\right\}$. Let $N(u)=g(u)+h(x, t)$ and observe that $\|N(u)\|_{L^{2}(\Omega)} \leqslant\|g(u)\|+\|h\|$. But

$$
\int_{\Omega}|g(u)|^{2} d x d t \leqslant \int_{\Omega}(A(u)+B)^{2} d x d t \leqslant A^{2}\|u\|^{2}+2 A B|\Omega|^{1 / 2}\|u\|+B^{2}|\Omega|,
$$

where $|\Omega|$ denotes the measure of $\Omega$. Now choose $R_{0}$ so large that $R>R_{0}$ implies that

$$
\left(A^{2} R^{2}+2 A B|\Omega|^{1 / 2} R+B^{2}|\Omega|\right)^{1 / 2}+\|h\| \leqslant R,
$$

which is clearly possible since $A<1$. We may now conclude that the nonlinear map $u \rightarrow L^{-1}(g(u)+h(x, t))$ maps the ball $B_{R}$ into itself. Since, in addition, by virtue of (i) the map is a (nonstrict) contraction, we may deduce the existence of a fixed point $u_{0} \in B_{R}$ where

$$
u_{0}=L^{-1}\left(g\left(u_{0}\right)+h(x, t)\right),
$$

and thus the existence of a weak solution to (2).

Question 1. In view of the recent result of Bahri and Brezis [1] on a LandesmanLazer theorem with infinite-dimensional kernel, it seems reasonable to suspect that if $h \perp \operatorname{ker}\{L-I\}$, then there exists a solution to (2) if $g=u+\arctan u$. 
THEOREM 2. Let $\sigma(L)=\left\{a_{n}\right\}_{-\infty}^{\infty}$ where $a_{n}<a_{n+1}$. Assume that

$$
\begin{gathered}
\left|g(x)-g(y)-\left(\frac{a_{n+1}+a_{n}}{2}\right)(x-y)\right| \leqslant \frac{\left|a_{n+1}+a_{n}\right|}{2}|x-y|, \\
\left|g(x)-\left(\frac{a_{n+1}+a_{n}}{2}\right) x\right| \leqslant A|x|+B
\end{gathered}
$$

for some constants, $A, B, 0<A<\left(a_{n+1}-a_{n}\right) / 2$. Then equation (2) has a solution for all $h \in L^{2}(\Omega)$.

Proof. Rewrite (2) as

$$
L u-\left(\frac{a_{n+1}+a_{n}}{2}\right) u=g(u)-\left(\frac{a_{n+1}+a_{n}}{2}\right) u+h(x, t)
$$

and observe that

$$
\left\|\left(L-\left(\frac{a_{n+1}+a_{n}}{2}\right) I\right)^{-1}\right\|=\left(\frac{a_{n+1}-a_{n}}{2}\right)^{-1} .
$$

The rest of the proof proceeds along the lines of Theorem 1.

REMARK 1. If, in particular, $a_{n} \leqslant g^{\prime}(u) \leqslant a_{n+1}$ and $a_{n}<g^{\prime}( \pm \infty)<a_{n+1}$, it is easy to check that hypotheses (i) and (ii) are satisfied.

REMARK 2. If the inequality in hypotheses (ii) is replaced by strict inequality, the solution is unique.

REMARK 3. Similar theorems apply if, instead of solutions of period $\sqrt{2 \pi}$, we search for solutions of period $2 \pi \sqrt{m / n}$, where $m, n$ are square free. In this case the eigenvalues would be $j^{2}-m k^{2} / n$ of infinite multiplicity.

3. More general periods. In this section we deal with the case where the period $T=2 \pi / \alpha$, where $\alpha \neq \sqrt{n}$ for any integer $n$. In this case the spectrum is the closure of the eigenvalues $j^{2}-\alpha^{2} k^{2}$ with the associated eigenfunctions

$$
\{\sin j x \cos \alpha k t, \sin j x \sin \alpha k t\} \text {. }
$$

In order to study the set $j^{2}-\alpha^{2} k^{2}$ for general $\alpha$, we need the concept of the continued fraction expansion of the number $\alpha$. This idea is useful in describing how well the irrational number $\alpha$ can be approximated by a rational number. Then, since

$$
j^{2}-\alpha^{2} k^{2}=k^{-2}(j / k-\alpha)(j / k+\alpha),
$$

we shall be able to give lower bounds for $j^{2}-\alpha^{2} k^{2}$ if we know that $|\alpha / k-\alpha| \geqslant \varepsilon / k$.

We present here the essential properties of the theory of continued fractions. The reader is referred to [9] for more details. For $n=0,1,2, \ldots$, let $a_{n}$ denote an integer and assume $a_{n}>1$, for $n=1,2, \ldots$. Define the quantity $\left[a_{0}, a_{1}, \ldots, a_{n}\right]$ inductively by setting $\left[a_{0}, a_{1}, \ldots, a_{n}\right]=p_{n} / q_{n}$, where $p_{-1}=1, q_{-1}=0, p_{0}=q_{0}, g_{0}=1, p_{1}=p_{-1}$ $+a_{1} p_{0}, q_{1}=q_{-1}+a_{1} q_{0}$ or, in general,

$$
p_{n+1}=p_{n-1}+a_{n+1} p_{n}, \quad q_{n+1}=q_{n-1}+a_{n+1} q_{n}
$$

for $n=0,1,2, \ldots$ For example, $[0,1,2,3]=7 / 10$ and if $\alpha=(1+\sqrt{5}) / 2$, then $\alpha=[1,1,1, \ldots]$. 
Every irrational number $\alpha$ can be represented as a continued fraction $\alpha=$ $\left[a_{0}, a_{1}, \ldots\right]=\lim _{n \rightarrow \infty}\left[a_{0}, \ldots, a_{n}\right]$ where the $a_{n}$ are integers with $a_{n} \geqslant 1$ for $n=$ $1,2, \ldots$ Conversely, given any collection of integers $a_{n}, n=0,1, \ldots$, with $a_{n} \geqslant 1$ for $n=1,2, \ldots$, then $\lim _{n \rightarrow \infty}\left[a_{0}, a_{1}, \ldots, a_{n}\right]$ exists and is an irrational number $\alpha$.

The two properties we use are the following:

Proposition 1. If $\alpha=\left[a_{0}, a_{1}, \ldots\right]$ and $|\alpha-p / q|<1 / 2 q^{2}$ for some rational number $p / q$, then $p / q=p_{n} / q_{n}$ for some $n=0,1,2, \ldots$

Proposition 2. For $n=0,1,2, \ldots$ one has

$$
\frac{1}{q_{n}^{2}\left(a_{n+1}+2\right)} \leqslant\left|\alpha-\frac{p_{n}}{q_{n}}\right| \leqslant \frac{1}{q_{n}^{2} a_{n+1}} .
$$

Thus, Propositions 1 and 2 tell us that if $\alpha=\left[a_{0}, a_{1} \ldots\right]$ with $a_{n} \leqslant M$ for some $M$, then there exists $\varepsilon$ such that $|j / k-\alpha| \geqslant \varepsilon / k^{2}$, and thus $j^{2}-\alpha^{2} k^{2} \geqslant \varepsilon$.

Actually, it tells us a little more. It tells us that if $\alpha=(1+\sqrt{5}) / 2$, then by a routine calculation, since $a_{n+1}=1$, we have $|\alpha-j / k| \geqslant 1 / 3 k^{2}$ and, thus, $\mid j^{2}-$ $\alpha^{2} k^{2} \mid \geqslant \alpha / 3$. Note that Proposition 2 gives, for some $\varepsilon>0$,

$$
\left|p_{n}^{2}-\alpha^{2} q_{n}^{2}\right|=\left|\frac{1}{q_{n}^{2}}\left(\frac{p_{n}}{q_{n}}-\alpha\right)\left(\frac{p_{n}}{q_{n}}+\alpha\right)\right| \leqslant 2 \alpha+\varepsilon,
$$

and thus we know that the spectrum has a point of accumulation in the interval $[-2 \alpha, 2 \alpha]$.

One can also check that since $p_{n} / q_{n}=\left[a_{0}, a_{1}, \ldots, a_{n}\right]$ is the best rational approximation of $\alpha$, then if the continued fraction expansion of $\alpha$ contains every prescribed finite block of integers infinitely often, then $j^{2}-\alpha^{2} k^{2}$ is dense in $\mathbf{R}^{1}$. This happens for almost all irrational numbers $\alpha$. In this case problem (3) will not have solutions for all $h$ even for linear $g$, so for the remainder of this section we confine ourselves to the case where $\alpha$ has a bounded continued fraction expansion. In this case our first theorem is

THEOREM 3. Let $\alpha$ have bounded continued fraction expansion. Then there exists $\varepsilon>0$ such that if $\left|g^{\prime}(s)\right|<\varepsilon$ for all $s \in R$, the equation

$$
\begin{gathered}
u_{t t}-u_{x x}-g(u)=h(x, t) \quad 0<x<\pi, \\
u(0, t)=u(\pi, t)=0, \quad u(x, t+2 \pi / \alpha)=u(x, t),
\end{gathered}
$$

has a unique weak solution for all $h \in L^{2}([0, \pi] \times[0,2 \pi / \alpha])$.

Proof. This is almost identical to the proofs of Theorems 1 and 2. We have only to observe that there exists $\varepsilon^{1}>0$ such that $\sigma(L) \cap\left(-\varepsilon^{1}, \varepsilon^{1}\right)=\varnothing$, and then choose $\varepsilon<\varepsilon^{1}$. Then the map $u \rightarrow L^{-1}(g(u)+h(x, t))$ is a strict contraction on the Hilbert space $L^{2}$ and has a fixed point. The proof is complete.

We now present a more general theorem, the proof of which is only sketched, as the hypotheses are somewhat difficult to verify.

We call $\sigma_{\mathrm{fr}}(L)$ the set of all points $x$ in the real line which are isolated eigenvalues of finite multiplicity. The remainder of the spectrum is called $\sigma_{\text {ess }}(L)$. Without loss of generality, we assume that $-\infty<g^{\prime}(-\infty) \leqslant g^{\prime}(+\infty)<\infty$. 
Theorem 4. Assume $\left[g^{\prime}(-\infty), g^{\prime}(+\infty)\right] \cap \sigma(L)=\varnothing$ and $\operatorname{Range}\left(g^{\prime}(s) \cap \sigma_{\mathrm{ess}}(L)\right)$ $=\varnothing$. Then equation (3) has a solution for all $h(x, t)$.

Proof. The proof follows along fairly standard lines. Let $H_{1}$ be the space spanned by the orthogonal eigenfunctions in the set $\sigma_{\text {ess }}(L)$. Let $P$ be orthogonal projection onto $H_{1}$. Choose $\alpha \in\left[g^{\prime}(-\infty), g^{\prime}(+\infty)\right]$ so that

$$
\operatorname{dist}\left(\alpha,\left\{g^{\prime}(-\infty), g^{\prime}(+\infty)\right\}\right)<\operatorname{dist}(\alpha, \sigma(L)) .
$$

Then one easily checks that the map

$$
u \rightarrow(L-a)^{-1}(g(u)-\alpha(u+h(x, t)))
$$

maps every ball of radius $R \geqslant R_{0}$ (centered at the origin) into itself for $R_{0}$ sufficiently large.

Now let $h=h_{1}+h_{2}$ where $h_{1} \in P H, h_{2} \in(I-P) h$. Then the equation $L u=$ $g(u)+h$ is equivalent to, for any $\beta \notin \sigma(L)$,

$$
\begin{aligned}
& (I-P)(L u-\beta u)=(I-P)\left(g(u)-\beta(u)+h_{2}\right), \\
& P(L u-\beta u)=P\left(g(u)-\beta u+h_{1}\right) .
\end{aligned}
$$

Let $u=v+w$ where $v \in(I-P) H, w \in P H$. Then (4) is equivalent to

$$
\begin{aligned}
& \text { (i) } v=(L-\beta)^{-1}(I-P)\left(g(v+w)-\beta v+h_{2}\right), \\
& \text { (ii) } w=(L-\beta)^{-1} P\left(g(v+w)-\beta w+h_{1}\right) .
\end{aligned}
$$

Now choose $\beta \notin \sigma(L)$ such that $\beta \in$ Range $g^{\prime}(s)$ and

$$
\operatorname{dist}\left(\beta, \text { bdry }\left(\text { Range } g^{\prime}\right)\right)<\operatorname{dist}\left(\beta, \sigma_{\text {ess }}(L)\right) .
$$

Then the map $T: H_{1} \rightarrow H_{1}$, defined by

$$
T w=(L-\beta)^{-1} P\left(g(v+w)-\beta w+h_{1}\right),
$$

is a strict contraction, and thus for each $v \in(I-P) H$, there exists a unique $w(v) \in P H$ which is a solution of (5)(ii). Moreover, $w$ is Lipschitz continuous with respect to $v$. Thus we have only to show that there exists a $v \in(I-P) H$ such that

$$
v=(L-\beta)^{-1}(I-P)\left(g(v+w)-\beta v+h_{2}\right) .
$$

However, (6) has a solution if and only if

$$
v=(L-\alpha)^{-1}(I-P)\left(g(v+w)-\alpha v+h_{2}\right)
$$

has a solution. On the other hand, we know that if $\|v\| \leqslant R>R_{0}$, then

$$
\begin{aligned}
\|v+w(v)\|^{2} \geqslant & \left\|(L-\alpha)^{-1}(I-P)\left(g(v+w(v))-\alpha v+h_{2}\right)\right\|^{2} \\
& +\left\|(L-\alpha)^{-1} P\left(g(v+w(v))-\alpha w+h_{1}\right)\right\|^{2}
\end{aligned}
$$

and since

$$
\|w(v)\|=\left\|(L-\alpha)^{-1} P\left(g(v+w(v))-\alpha w(v)+h_{1}\right)\right\|,
$$


equation (7) implies that

$$
\|v\|^{2} \geqslant\left\|(L-\alpha)^{-1}(I-P)\left(g(v+w(v))-\alpha v+h_{2}\right)\right\|^{2} .
$$

This implies that the compact map

$$
T v=(L-\alpha)^{-1}(I-P)\left(g(v+w(v))-\alpha v+h_{2}\right)
$$

maps the ball of radius $R$ into itself and thus has a fixed point and (6) has a solution. This proves the theorem.

\section{REFERENCES}

1. A. Bahri and H. Brezis, Periodic solutions of a nonlinear wave equation, Proc. Roy. Soc. Edinburgh Sect. A 85 (1980), 313-320.

2. H. Brezis and L. Nirenberg, Characterization of the ranges of some nonlinear operators, and applications to boundary value problems, Ann. Scuola Norm. Pisa Cl. Sci. 5 (1978), 225-326.

3. H. Brezis, Periodic solutions of nonlinear vibrating strings, Proc. Sympos. Pure Math., vol. 39, Amer. Math. Soc., Providence, R. I., 1983.

4. J. Cassels, An introduction to Diophantine approximation, Cambridge Univ. Press, Cambridge, 1965.

5. A. C. Lazer, Application of a lemma on bilinear forms to a problem in nonlinear oscillations, Proc. Amer. Math. Soc. 33 (1972), 89-94.

6. P. J. McKenna, On the reduction of a semilinear hyperbolic problem to a Landesman-Lazer problem, Houston J. Math. 4 (1978), 577-581.

7. I. Niven and H. S. Zuckerman, The theory of numbers, 4 th ed., Wiley, New York, 1980.

8. P. H. Rabinowitz, Some minimax theorems and applications to nonlinear partial differential equations, Nonlinear Analysis, a volume in honor of E. H. Rothe, Academic Press, 1976.

9. G. R. Sell, The prodigal integral, Amer. Math. Monthly 84 (1977), 162-167.

Department of Mathematics, University of Florida, Gainesville, Florida 32611 